\title{
Getting ready for GeMS 2.0: A workhorse AO facility
}

\author{
Gaetano Sivo ${ }^{a}{ }^{*}$, Eduardo Marin ${ }^{a}$, Vincent Garrel $^{b}$, Benoit Neichel ${ }^{c}$, Marcos van Dam ${ }^{d}$, \\ François Rigaut ${ }^{e}$, Cristian Moreno $^{a}$, Emmanuel Chirre ${ }^{a}$, Gabriel Perez $^{a}$, Angelic Ebbers ${ }^{a}$, Paul \\ Collins $^{a}$, Vicente Vergara ${ }^{a}$, Eduardo Toro ${ }^{a}$, Pablo Diaz ${ }^{a}$, Brian Chinn ${ }^{a}$, Carlos Figueroa ${ }^{a}$, Jeff \\ Donahue $^{f}$, Pedro Gigoux ${ }^{a}$, Ian Price ${ }^{e}$, Nick Herrald ${ }^{e}$, Francis Bennet ${ }^{e}$, Rodrigo Carrasco ${ }^{a}$, \\ Morten Andersen ${ }^{a}$, Rodolfo Angeloni ${ }^{g}$, Manuel Lazo ${ }^{a}$, Vanessa Montes ${ }^{a}$, Rene Rutten ${ }^{a}$, Céline \\ d'Orgeville ${ }^{e}$, Paul Hirst ${ }^{f}$ \\ ${ }^{a}$ Gemini South Observatory, casilla 603, La Serena, Chile; \\ ${ }^{b}$ Max Planck Institut fuer extraterrestrische Physik, 85748, Garching, Germany; \\ ${ }^{c}$ Laboratoire d'Astrophysique de Marseille, Université Aix-Marseille, UMR7326, CNRS-INSU, \\ Marseille, France; \\ $d$, Flat Wavefronts, 21 Lascelles street, Cristchurch 8022, New Zealand; \\ ${ }^{e}$ Australian National University, Research School of Astronomy and Astrophysics, Mount \\ Stromlo Observatory, Cotter Road, Weston Creek, ACT 2611, Australia; \\ ${ }^{f}$ Gemini North Observatory, 670 N. A'ohoku place, Hilo, HI, United States of America. \\ ${ }^{g}$ Departamento de Fisica y Astronomia, Universidad de La Serena, Avenida Juan Cisternas \\ 1200 Norte, La Serena, Chile
}

\begin{abstract}
AO systems aim at detecting and correcting for optical distortions induced by atmospheric turbulences. The Gemini South telescope has currently two main AO systems: the Gemini Multi Conjugated AO System GeMS and the Gemini Planet Imager GPI. GeMS is operational and regularly used for science observation delivering close to diffraction limit resolution over a large field of view $\left(85 \times 85 \operatorname{arcsec}^{2}\right)$. We first review the performance obtained this past year. Then we will go in the details of the strong modifications GeMS is currently having. We are integrating a new laser system in the laser guide star facility module. We are also advancing in the development of the new Natural Guide Star wavefront sensor. We present as well in this paper the preliminary results obtained with our new installed NGS mask that allows us better astrometric precision.
\end{abstract}

Keywords: Adaptive Optics, Multi-Conjugated Adaptive Optics, GeMS, astrometry, Laser Guide Star

\section{INTRODUCTION}

GeMS, ${ }^{1,2}$ the Gemini Multi Conjugated Adaptive Optics (AO) System is a Multi Conjugated AO (MCAO) facility installed at the Cassegrain focus of the $8.1 \mathrm{~m}$ Gemini South telescope at the top of Cerro Pachón (Chile). GeMS delivers a uniform, close to diffraction limit, corrected image in the near infrared (from $0.95 \mu m$ to $2.5 \mu \mathrm{m}$ ) over a wide field of view (FoV) of 2'. GeMS is the first sodium laser assisted MCAO instrument and is currently still the only one in regular operation. GeMS uses 5 Laser Guide Stars (LGSs) placed on a 60" diameter squared constellation with one star in the center. The LGS light goes then to five $16 \times 16$ Shack-Hartmann wavefront sensor to measure the distorted wavefront. These measurements are then used to drive two deformable mirrors, one conjugated to the ground, one conjugated at $9 \mathrm{~km}$ at a speed up to $800 \mathrm{~Hz}$ (depending on the photon return). To correct for tip-tilt (TT) errors, GeMS uses up to three Natural Guide Stars (NGSs) using a second set of wavefront sensor. Currently GeMS has three probes that can move in the technical FoV in order to catch the light from the NGS and sense the TT. One of these probes has also a second funcionality, it sends $30 \%$ of the light into a Slow Focus Sensor (SFS) in order to compensate the slow altitude drift of the sodium layer altitude

Further author information: (Send correspondence to Gaetano Sivo)

*Gaetano Sivo: E-mail: gsivo@gemini.edu, Telephone: +56 512205642 
in the mesosphere. The magnitude limit for this NGSWFS system is about 15.5 in V-band. We are currently developing with the Australian National University a new more reliable NGSWFS (see section 4).

Since GeMS is in operation, it has been combined with the $85^{\prime \prime 2}$ near infrared imager GSAOI. ${ }^{3}$ GSAOI has 4 $2048 \times 2048$ pixels Hawaii-2RG and is equipped with a decent number of wide and narrow band filters. GeMS, being an AO facility, could also be used with other facilities instruments available at Gemini South, such as Flamingos- $2^{4}$ (a near infrared spectro-imager) or GMOS-S ${ }^{5}$ (a multi-objects spectrograph in the visible).

\section{PERFORMANCE STATUS OF GEMS AND SCIENCE EXAMPLES}

GeMS performance have been steady since commissioning and under good seeing conditions can reach $40 \%$ SR in K-band all over the science FoV (see Fig 1).

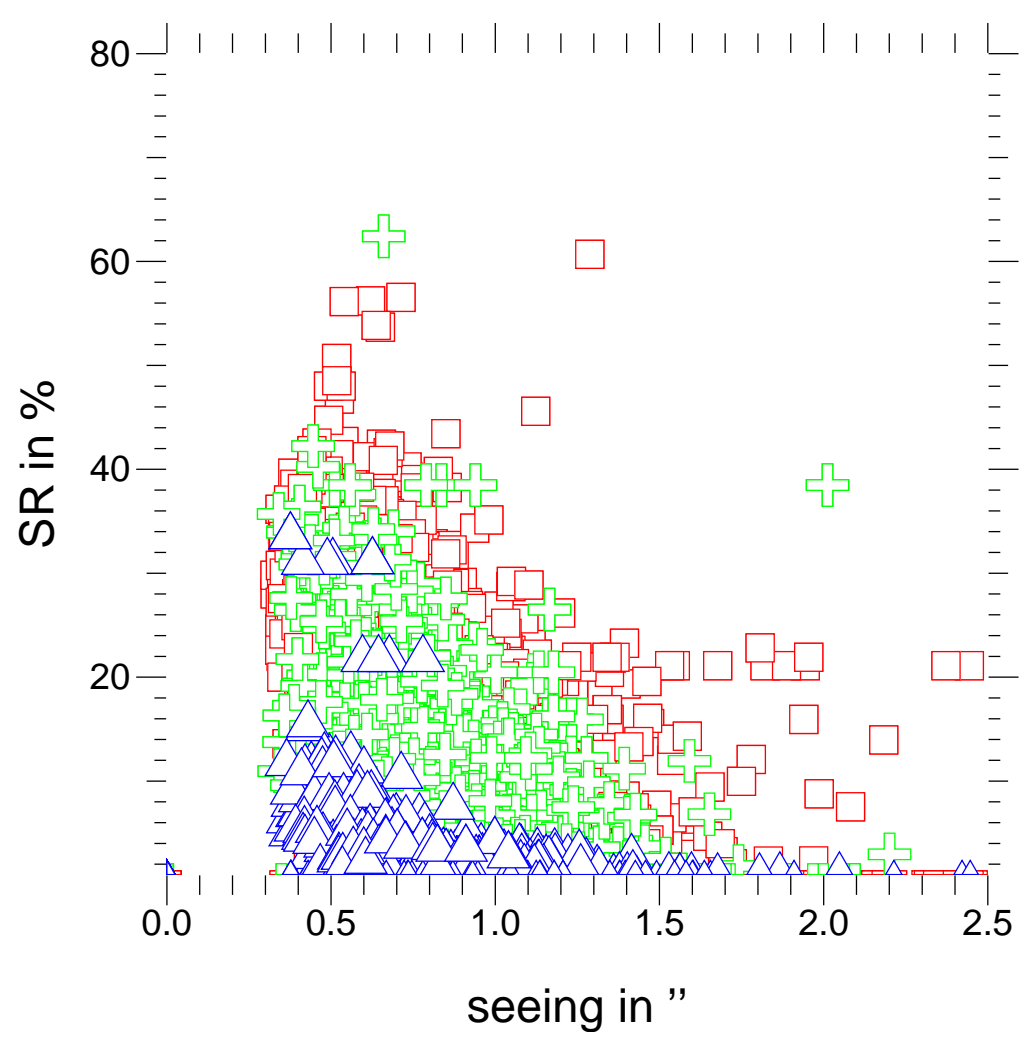

Figure 1. Performance obtained with GeMS during the period 2014-2017 in term of Strehl ratio as a function of the seeing condition (Red: K-band. Green: H-band. Blue: J-band)

Maintaining good performance has been more challenging under medium to bad seeing conditions mainly due to the lack of power of our laser system. To overcome this issue, we decided to replace our laser system with a new, of the shelf, more reliable laser: the SodiumStar $22 \mathrm{~W}$ Toptica laser ${ }^{6}$ (see section 3 ). Note that the same laser has been installed at Paranal for the $\mathrm{VLT}^{7}$ and at Mauna Kea for Keck. ${ }^{8}$

Science programs with GeMS GSAOI covers a very wide field of astrophysics. The coupled system GeMS GSAOI, delivering high spatial resolution over a wide FoV has allowed several team to reach unprecedented depth in ground based photometric observations of crowded fields such as stellar population. ${ }^{9-11}$ It also helped to push the study of stellar population and formation in very different environment than our own galaxy, uncovering several hundreds of Young Stellar Objects candidates in the Large Magellanic Cloud ${ }^{12}$

Finally, during the GeMS commissioning, observations have also been done using GMOS-S in its imaging mode. The experiment was successful. The performance obtained were better than an equivalent Ground Layer AO corrections in the visible, i.e. the Full Width Half Maximum was improved by a factor 2 compared to median seeing limited observations. More details can be found in these papers. ${ }^{13,14}$ 


\section{A NEW LASER FACILITY: THE TOPTICA SODIUMSTAR20/2}

The laser subsystem is one of the most important components of the full GeMS facility. Our current laser, a $50 \mathrm{~W}$ solid-state laser delivered by Lockheed Martin Coherent Technologies (LMCT) in March 2010, has seen its first light on-sky in January 2011. ${ }^{15}$ Two infrared laser arms are independently amplified several times before being mixed into a sum frequency generation birefringent crystal to produce a laser line centered at the D2a sodium resonance. This very complex system is located inside a container box mounted on the side of the telescope platform providing a control environment in a gravity invariant area. Since its first light in 2011, a very non negligible amount of staff has been required to keep the system in a working state for operations. The laser power has been very unstable and rarely at nominal working value. The figure 2 shows the output laser power as a function of time. We can see than since 2012, the laser keeps dropping and this affects directly the performance achievable with GeMS.



Figure 2. Output laser power registered as a function of time.

In 2014, we started to work internally at Gemini, to see if it was possible to find a replacement for this laser system that would allow us to reach the performance level needed for our science but also being a much more reliable laser. The main requirements were delivering a similar photon return but with an increased robustness and ease of use in order to reduce significantly the man power internally and the engineering workload and provide a sustainable long term operation for GeMS. Based on a successful internal feasibility study within the AO team, the Gemini board endorsed the procurement of a new laser system. The procurement phase was done via a public request for quotes and after reviewing the answers, the internal committee with the help of 2 external reviewers, have decided to select the Toptica SodiumStar 20/2 laser. ${ }^{6}$ In 2017, the laser has been tested in the lab, and integrated on the telescope. The commissioning of this new laser with GeMS is scheduled for the last week of October 2017. We do not show results of the commissioning in this paper yet, but we present hereafter the small modifications needed for the integration. These modification are mainly due to the fact that we do not want to remove the current LMCT laser yet. We thus need to integrate a Beam Injector Module (BIM) and have a new safety system in our Laser Interlock System (LIS) to be able to work with both configuration. We will of course never use at the same time both laser, but during the commissioning we intend to do a direct on-sky study of using on-sky for GeMS two completely different technologies of Sodium lasers. We will be able to compare the physical properties and understand maybe better the sodium layer in the mesosphere.

We decided to install the new laser on the same laser platform as the LMCT, on the side of the current LMCT laser box, and modify slightly the injection of the laser into our current unmodified Beam Transfer Optics (BTO) (see figure 3). 


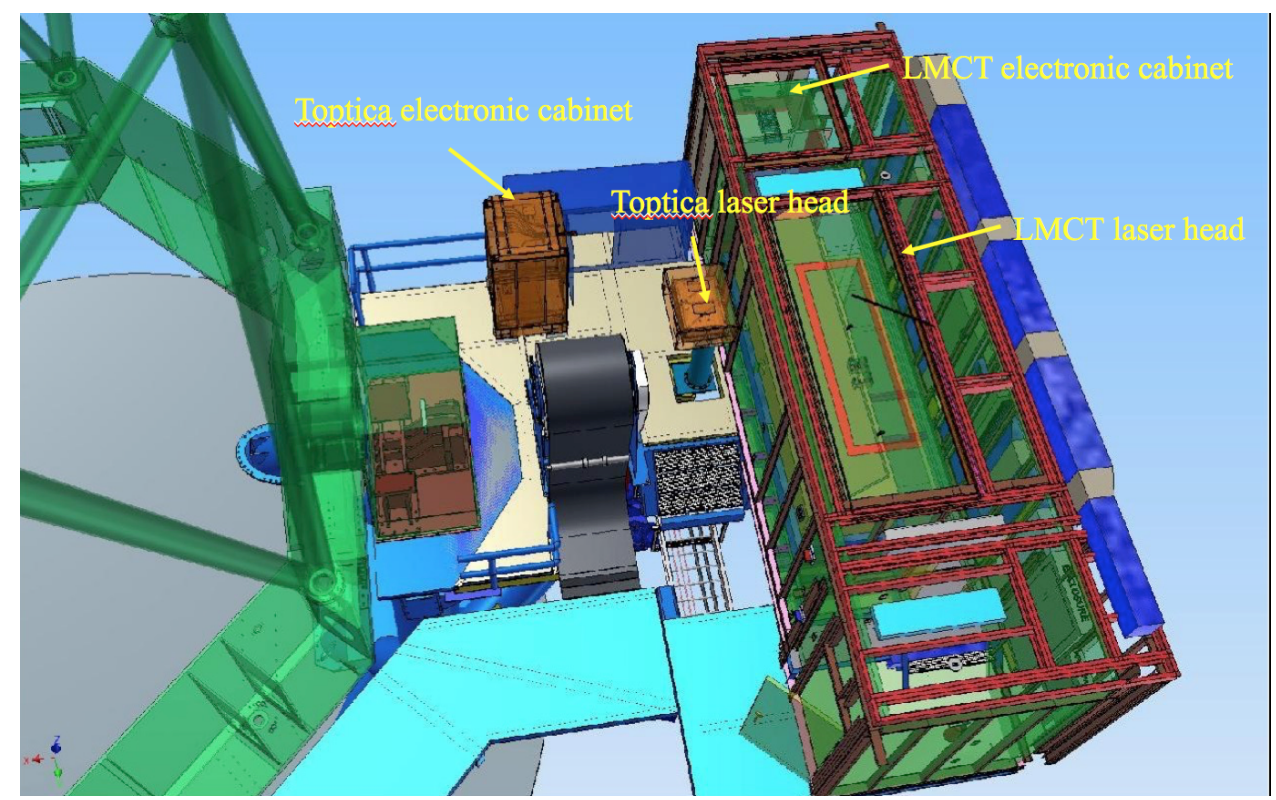

Figure 3. Drawing showing the laser platform and the current location of the LMCT and the Toptica laser. We can see the order of magnitude difference in size for these 2 lasers.

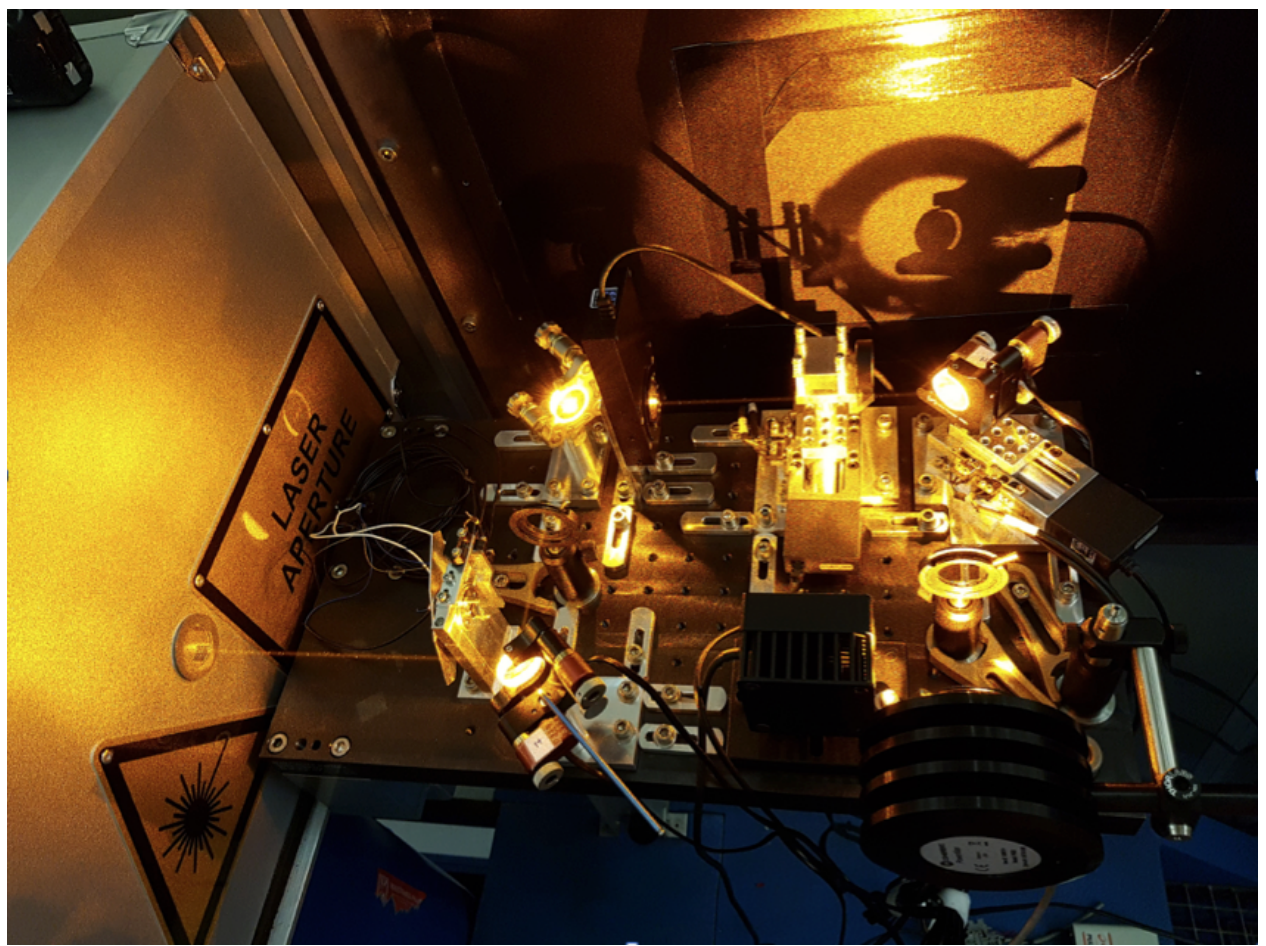

Figure 4. Beam injector module installed between the exit shutter of the laser Toptica laser, the exit shutter of the LMCT and the entrance torque tube of the BTO.

\section{NGS2: THE NEXT GENERATION SENSOR FOR NATURAL GUIDE STAR FOR GEMS}

GeMS uses 5 LGSs for high order wavefront sensing and up to three NGSs for TT and plate scale modes sensing. The current NGS WFS inside GeMS is based on a complicated, difficult to align optical setup and suffers from 
very high loss in throughput therefore decreasing strongly the limiting magnitude by approximately 2.5 which gives an overall working sky coverage for GeMS of less than 5\% rather than the original specification of $30 \%$. An another strong limitations is related to field distortions. The GeMS AO bench called CANOPUS is based on a two $F / 16-F / 16$ off axis parabola optical design to feed the LGSWFS. This introduces significant geometrical distortions in the focal plane. As a consequence of the present distortions, an NGS asterism will change shape when it is offsetted across the output focal plane. The current NGS WFS based on probes that move into the technical FoV do not follow these distortions, when the offsets are becoming significant, it is sure than the probes will not arrive to the star position and we will need a new acquisition phase. The working value offset is about 10". An illustration of the distortions induced by the AO bench is illustrated in the figure 5 . The overhead introduced for this is quite large. Due to all these issues, it has been decided to replace as well the current NGS WFS with a more reliable system delivering better throughput, better limiting magnitude and ease of use during operation.



Figure 5. CANOPUS science focal plane distortions. the pixel scale is 20 mas. This map was obtained on a well-known astrometric field ${ }^{16}$

In collaboration with the Australian National University (ANU), we have been working on a new focal plane NGS WFS. The idea is based a single focal plane array to do multiple TT wavefront sensing. The system is called NGS2. ${ }^{17}$ This focal plane array uses multi region of interests to read fast rates windows up to $800 \mathrm{~Hz}$ with a very low read out noise of about $0.3 e^{-}$. The performance in simulations are promising showing good performance in medium seeing conditions using NGS of about 18th magnitude. This will bring back the required sky coverage 
for GeMS (results shown in figure 6).
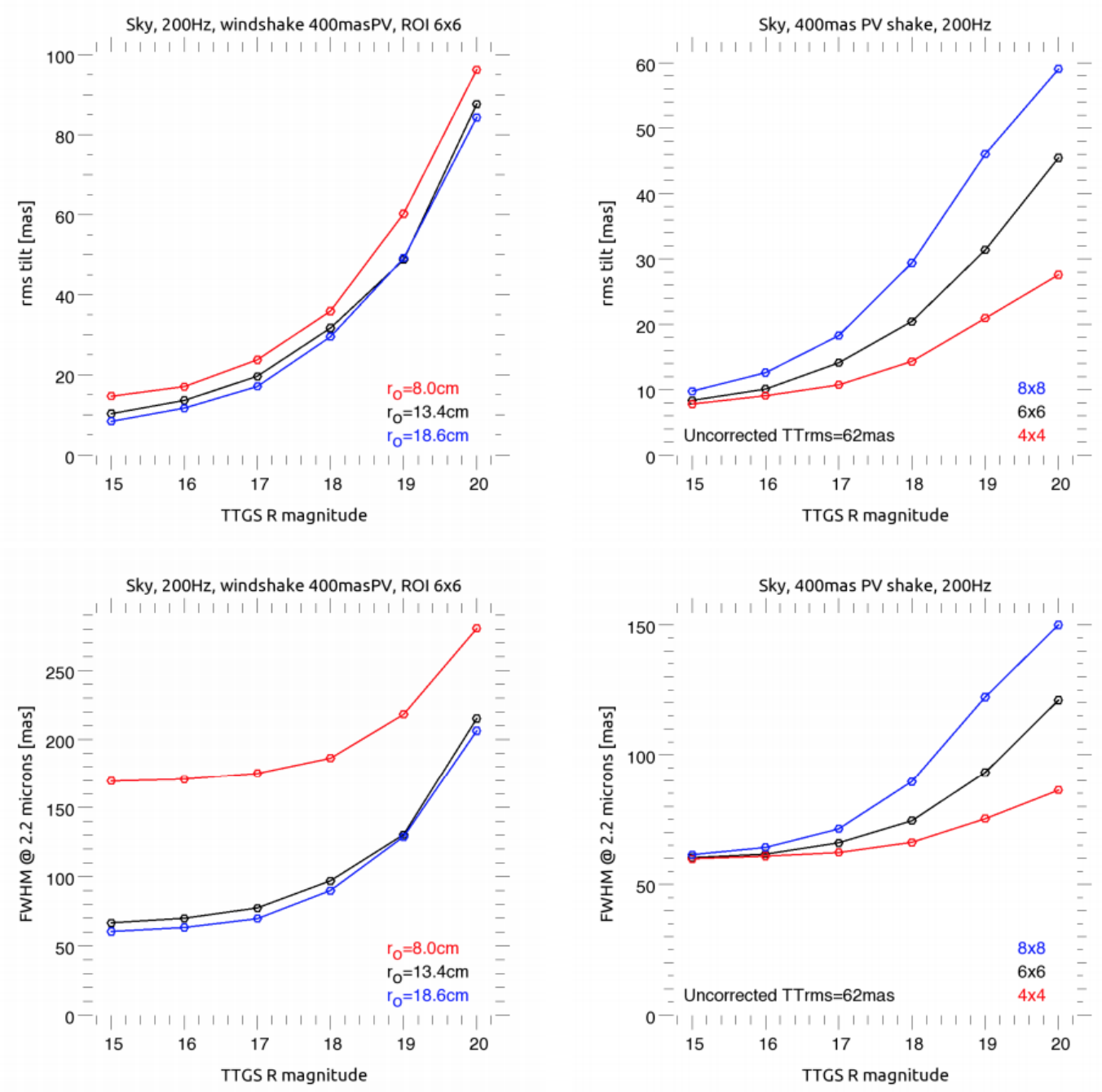

Figure 6. NGS2 performance results obtained on simulation using YAO. Top: Tilt RMS as a function of the guide star magnitude in $\mathrm{R}$ for 3 different seeing conditions at 500nm. Bottom: Same simulations but showing the FWHM on the image in K-band as a function of the guide star magnitude in R. The high order correction is taken into account using nominal rms correction of about 47 mas.

Replacing the current NGS WFS, will also impact our SFS subsystem. It is indeed currently embedded into the NGS WFS. With NGS2 we will need an another way to measure the slow focus offset due to the conjugation of the sodium layer altitude. For that we will use one of the peripheral WFS of Gemini (P1WFS) to measure this. P1WFS has a large patrol field, so in order to not vignette with the CANOPUS technical FoV, P1WFS will catch a star up to 7' away and use this to measure the change in focus. More details can be found in the proceeding here ${ }^{18}$ from this conference.

\section{ASTROMETRIC CALIBRATION}

Although astrometry was not a requirements during the GeMS design phases, during GeMS operations it has been shown that astrometry can be a serious candidate in the possible science cases. Several science papers have been published in this area..$^{9,10,19-21}$ The astrometric precision is currently limited by some undetermined dynamic 
systematic effects to about 0.4 to 0.5 mas rms after calibration of high-order distortions in post-processing. ${ }^{19}$ This performance can currently be achieved only on crowded fields, meaning we need a decent number of stars in each quadrant of GSAOI. Calibrating and understanding the sources of distortions requires an accurate reference target that can be inserted in the Canopus bench at any time. We did in 2016, add a calibrating mask in the focal plane calibration source to help $\mathrm{us}^{22}$ (see figure 7 ). The mask is a custom mask designed by quartz photomask covered in chrome with sub-micron precision on the position and size of clear pinholes and multiply their number so they can cover the entire CANOPUS FoV with a pitch of $1.19255 \mathrm{~mm}$. Additionally empty spaces have been left so we can still use our current Non Common Path Aberrations (NCPA) procedure to calibrate them. ${ }^{23}$

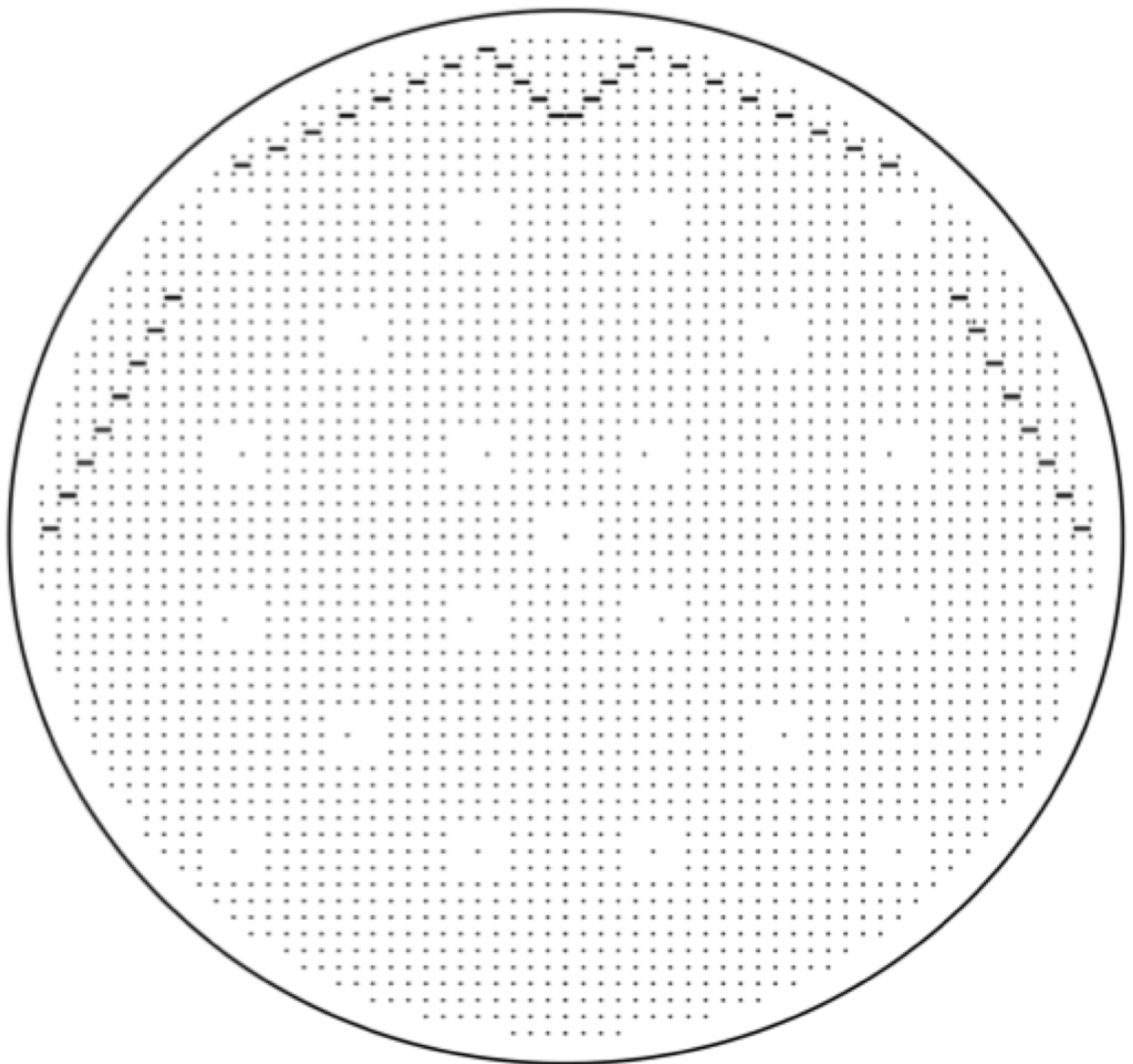

Figure 7. Schematic of the diffractive mask installed to calibrate field distortions inside CANOPUS.

On a longer term, for science targets in sparse fields, a diffractive pupil mask, ${ }^{24}$ carefully creating diffraction spikes in the image is forecasted. In sparser fields, the astrometry is particularly interesting for brown dwarfs and possibly for exoplanets detection through astrometric measurements. We note as well that there are also an important effort in the community to work on numerical methods as well to correct for field distortions introduced during AO observations. A recent paper explained very clearly such a technique. ${ }^{25}$ 


\section{CONCLUSION}

GeMS is in operation and delivering very good new exciting science since 2012. While we are still in operation and working on maintaining the system, we are actively working on strong upgrades that will bring GeMS into a more reliable system and helping our community to reach for the first time resolution on several important science cases. The new laser will improve the reliability of GeMS operations. NGS2 will open new exciting science cases for GeMS to the Gemini community thanks to its high improvement in term of sky coverage. GeMS is not only evolving for better operations, it is also evolving for better data quality. Thanks to the previous upgrades mentioned, we will reach better stability in term of performance. PSF reconstructions may be easier. The new focal plane mask installed will help as well a lot the users to calibrate at least in the first order the field distortions induced by the AO bench. GeMS after its 5 years of operations is still an unique and complex laser assisted MCAO facility tackling efficiently science cases requiring both high spatial resolution and wide FoV. Reshaping GeMS will definitely bring the instrument as a worldwise recognised AO workhorse.

\section{ACKNOLEDMENTS}

Based on observations obtained at the Gemini Observatory, which is operated by the Association of Universities for Research in Astronomy, Inc., under a cooperative agreement with the NSF on behalf of the Gemini partnership: the National Science Foundation (United States of America), The National Research Council (Canada), CONICYT (Chile), the Australian Research Council (Australia), Ministério de Ciência, Tecnologia e Inovação (Brazil) and Ministerio de Ciencia, Tecnologia e Innovación Productiva (Argentina).

\section{REFERENCES}

[1] Rigaut, F., Neichel, B., Boccas, M., d'Orgeville, C., Vidal, F., van Dam, M. A., Arriagada, G., Fesquet, V., Galvez, R. L., Gausachs, G., Cavedoni, C., Ebbers, A. W., Karewicz, S., James, E., Lührs, J., Montes, V., Perez, G., Rambold, W. N., Rojas, R., Walker, S., Bec, M., Trancho, G., Sheehan, M., Irarrazaval, B., Boyer, C., Ellerbroek, B. L., Flicker, R., Gratadour, D., Garcia-Rissmann, A., and Daruich, F., "Gemini multiconjugate adaptive optics system review - I. Design, trade-offs and integration," MNRAS 437, 23612375 (Jan. 2014).

[2] Neichel, B., Rigaut, F., Vidal, F., van Dam, M. A., Garrel, V., Carrasco, E. R., Pessev, P., Winge, C., Boccas, M., d'Orgeville, C., Arriagada, G., Serio, A., Fesquet, V., Rambold, W. N., Lührs, J., Moreno, C., Gausachs, G., Galvez, R. L., Montes, V., Vucina, T. B., Marin, E., Urrutia, C., Lopez, A., Diggs, S. J., Marchant, C., Ebbers, A. W., Trujillo, C., Bec, M., Trancho, G., McGregor, P., Young, P. J., Colazo, F., and Edwards, M. L., "Gemini multiconjugate adaptive optics system review - II. Commissioning, operation and overall performance," MNRAS 440, 1002-1019 (May 2014).

[3] McGregor, P., Hart, J., Stevanovic, D., Bloxham, G., Jones, D., Van Harmelen, J., Griesbach, J., Dawson, M., Young, P., and Jarnyk, M. A., "Gemini South Adaptive Optics Imager (GSAOI)," in [Ground-based Instrumentation for Astronomy], Moorwood, A. F. M. and Iye, M., eds., Proc. SPIE 5492, 1033-1044 (Sept. 2004).

[4] Elston, R., Raines, S. N., Hanna, K. T., Hon, D. B., Julian, J., Horrobin, M., Harmer, C. F. W., and Epps, H. W., "Performance of the FLAMINGOS near-IR multi-object spectrometer and imager and plans for FLAMINGOS-2: a fully cryogenic near-IR MOS for Gemini South," in [Instrument Design and Performance for Optical/Infrared Ground-based Telescopes], Iye, M. and Moorwood, A. F. M., eds., Proc. SPIE 4841, 1611-1624 (Mar. 2003).

[5] Crampton, D., Fletcher, J. M., Jean, I., Murowinski, R. G., Szeto, K., Dickson, C. G., Hook, I., Laidlaw, K., Purkins, T., Allington-Smith, J. R., and Davies, R. L., "Gemini multi-object spectrograph GMOS: integration and tests," in [Optical and IR Telescope Instrumentation and Detectors], Iye, M. and Moorwood, A. F., eds., Proc. SPIE 4008, 114-122 (Aug. 2000).

[6] Enderlein, M., Friedenauer, A., Schwerdt, R., Rehme, P., Wei, D., Karpov, V., Ernstberger, B., Leisching, P., Clements, W. R. L., and Kaenders, W. G., "Series production of next-generation guide-star lasers at TOPTICA and MPBC," in [Adaptive Optics Systems IV], Proc. SPIE 9148, 914807 (July 2014).

[7] Bonaccini, D., "Laser Guide Star return flux at Cerro Paranal and at the Canary Islands," in [Fith International Conference on Adaptive Optics for Extremely Large Telescopes.], (2017). 
[8] Chin, J. C. Y., Wizinowich, P., Wetherell, E., Lilley, S., Cetre, S., Ragland, S., Medeiros, D., Tsubota, K., Doppmann, G., Otarola, A., and Wei, K., "Keck II laser guide star AO system and performance with the TOPTICA/MPBC laser," in [Adaptive Optics Systems V], Proc. SPIE 9909, 99090S (July 2016).

[9] Saracino, S., Dalessandro, E., Ferraro, F. R., Geisler, D., Mauro, F., Lanzoni, B., Origlia, L., Miocchi, P., Cohen, R. E., Villanova, S., and Moni Bidin, C., "Ultra-deep GEMINI Near-infrared Observations of the Bulge Globular Cluster NGC 6624.," Astrophys. Journal 832, 48 (Nov. 2016).

[10] Massari, D., Fiorentino, G., McConnachie, A., Bellini, A., Tolstoy, E., Turri, P., Andersen, D., Bono, G., Stetson, P. B., and Veran, J.-P., "Astrometry with MCAO: HST-GeMS proper motions in the globular cluster NGC 6681," Astronomy \& Astrophysics 595, L2 (Oct. 2016).

[11] Turri, P., McConnachie, A. W., Stetson, P. B., Fiorentino, G., Andersen, D. R., Véran, J.-P., and Bono, G., "Toward Precision Photometry for the ELT Era: The Double Subgiant Branch of NGC 1851 Observed with the Gemini/GeMS MCAO System," Astrophys. Journal Letters 811, L15 (Oct. 2015).

[12] Bernard, A., Neichel, B., Samal, M. R., Zavagno, A., Andersen, M., Evans, C. J., Plana, H., and Fusco, T., "Deep GeMS/GSAOI near-infrared observations of N159W in the Large Magellanic Cloud," Astronomy \& Astrophysics 592, A77 (July 2016).

[13] Hibon, P., Neichel, B., Garrel, V., Prout, B., Rigaut, F., Koning, A., Sivo, G., Gimeno, G., Carrasco, R., Winge, C., Pessev, P., Serio, A., and Arriagada, G., "First performance of the GeMS+GMOS system," in [Ground-based and Airborne Instrumentation for Astronomy V], Proc. SPIE 9147, 91478T (Aug. 2014).

[14] Hibon, P., Garrel, V., Neichel, B., Prout, B., Rigaut, F., Koning, A., Carrasco, E. R., Gimeno, G., and Pessev, P., "First performance of the GeMS + GMOS system - 1. Imaging," MNRAS 461, 507-518 (Sept. 2016).

[15] d'Orgeville, C., Diggs, S., Fesquet, V., Neichel, B., Rambold, W., Rigaut, F., Serio, A., Araya, C., Arriagada, G., Balladares, R., Bec, M., Boccas, M., Duran, C., Ebbers, A., Lopez, A., Marchant, C., Marin, E., Montes, V., Moreno, C., Petit Vega, E., Segura, C., Trancho, G., Trujillo, C., Urrutia, C., Veliz, P., and Vucina, T., "Gemini South multi-conjugate adaptive optics (GeMS) laser guide star facility on-sky performance results," in [Adaptive Optics Systems III], Proc. SPIE 8447, 84471Q (July 2012).

[16] Rigaut, F., Neichel, B., Boccas, M., d'Orgeville, C., Arriagada, G., Fesquet, V., Diggs, S. J., Marchant, C., Gausach, G., Rambold, W. N., Luhrs, J., Walker, S., Carrasco-Damele, E. R., Edwards, M. L., Pessev, P., Galvez, R. L., Vucina, T. B., Araya, C., Gutierrez, A., Ebbers, A. W., Serio, A., Moreno, C., Urrutia, C., Rogers, R., Rojas, R., Trujillo, C., Miller, B., Simons, D. A., Lopez, A., Montes, V., Diaz, H., Daruich, F., Colazo, F., Bec, M., Trancho, G., Sheehan, M., McGregor, P., Young, P. J., Doolan, M. C., van Harmelen, J., Ellerbroek, B. L., Gratadour, D., and Garcia-Rissmann, A., "GeMS: first on-sky results," in [Adaptive Optics Systems III], Proc. SPIE 8447, 84470I (July 2012).

[17] Rigaut, F., Price, I., d'Orgeville, C., Bennet, F., Herrald, N., Paulin, N., Uhlendorf, K., Garrel, V., Sivo, G., Montes, V., and Trujillo, C., "NGS2: a focal plane array upgrade for the GeMS multiple tip-tilt wavefront sensor," in [Adaptive Optics Systems V], Proc. SPIE 9909, 99091X (July 2016).

[18] Marin, E., Sivo, G., Garrel, V., Gigoux, P., Moreno, C., van Dam, M., Hirst, P., Montes, V., and Rutten, R., "Tracking the sadium layer altitude with GeMS in the era of NGS2," in [Fith International Conference on Adaptive Optics for Extremely Large Telescopes.], (2017).

[19] Neichel, B., Lu, J. R., Rigaut, F., Ammons, S. M., Carrasco, E. R., and Lassalle, E., "Astrometric performance of the Gemini multiconjugate adaptive optics system in crowded fields," MNRAS 445, 500-514 (Nov. 2014).

[20] Fritz, T. K., Linden, S. T., Zivick, P., Kallivayalil, N., Beaton, R. L., Bovy, J., Sales, L. V., Sohn, T., Angell, D., Boylan-Kolchin, M., Carrasco, E. R., Damke, G., Davies, R., Majewski, S., Neichel, B., and van der Marel, R., "The Proper Motion of Pyxis: The First Use of Adaptive Optics in Tandem with HST on a Faint Halo Object," Astrophys. Journal 840, 30 (May 2017).

[21] Garcia, E. V., Ammons, S. M., Salama, M., Crossfield, I., Bendek, E., Chilcote, J., Garrel, V., Graham, J. R., Kalas, P., Konopacky, Q., Lu, J. R., Macintosh, B., Marin, E., Marois, C., Nielsen, E., Neichel, B., Pham, D., De Rosa, R. J., Ryan, D. M., Service, M., and Sivo, G., "Individual, Model-independent Masses of the Closest Known Brown Dwarf Binary to the Sun," Astrophys. Journal 846, 97 (Sept. 2017). 
[22] Garrel, V., Sivo, G., Marin, E., Carrasco, R., Montes, V., Lazo, M., Gigoux, P., Moreno, C., Trujillo, C., Donahue, J., van Dam, M., Rigaut, F., d'Orgeville, C., Kulcsár, C., Juvénal, R., Araujo, C., Ammons, M., and Neichel, B., "Reshaping and polishing the GeMS MCAO system," in [Adaptive Optics Systems V], Proc. SPIE (2016).

[23] Gratadour, D., Rigaut, F., and Neichel, B., "Tomographic phase diversity for non-common path aberrations retrieval on wide field ao systems," 2, 2nd AO4ELT conference - Adaptative Optics for Extremely Large Telescopes (2011).

[24] Ammons, S. M., Bendek, E. A., Guyon, O., Macintosh, B., and Savransky, D., "Theoretical limits on bright star astrometry with multi-conjugate adaptive optics using a diffractive pupil," in [Adaptive Optics Systems III], Proc. SPIE 8447, 84470P (July 2012).

[25] Bernard, A., Neichel, B., Mugnier, L. M., and Fusco, T., "Optimal correction of distortion for High Angular Resolution images. Application to GeMS data," ArXiv e-prints (Sept. 2017). 